\title{
Corrigendum: Schisandrin B Antagonizes Cardiotoxicity Induced by Pirarubicin by Inhibiting Mitochondrial Permeability Transition Pore (mPTP) Opening and Decreasing Cardiomyocyte Apoptosis
}

\section{OPEN ACCESS}

Approved by: Frontiers Editorial Office

Frontiers Media SA, Switzerland

${ }^{*}$ Correspondence:

Peng Pu

pp841103@sina.com

Quan He

851557800th@sina.com

${ }^{+}$These authors have contributed

equally to this work

Specialty section:

This article was submitted to Cardiovascular and Smooth Muscle

Pharmacology,

a section of the journal

Frontiers in Pharmacology

Received: 17 October 2021

Accepted: 25 October 2021

Published: 11 November 2021

Citation:

Shi $H$, Tang $H$, Ai W, Zeng $Q$, Yang $H$, Zhu F, Wei Y, Feng R, Wen L, Pu P and $\mathrm{He} Q$ (2021) Corrigendum: Schisandrin B Antagonizes Cardiotoxicity Induced

by Pirarubicin by Inhibiting Mitochondrial Permeability Transition Pore (MPTP) Opening and Decreasing Cardiomyocyte Apoptosis. Front. Pharmacol. 12:796551. doi: 10.3389/fphar.2021.796551
Hongwei Shi ${ }^{1,2 \dagger}$, Heng Tang ${ }^{3 \dagger}$, Wen $\mathrm{Ai}^{4}$, Qingfu Zeng ${ }^{5}$, Hong Yang ${ }^{6}$, Fengqing Zhu ${ }^{3}$, Yunjie Wei ${ }^{7}$, Rui Feng ${ }^{3}$, Li Wen ${ }^{3}$, Peng $\mathrm{Pu}^{3 *}$ and Quan $\mathrm{He}^{3 *}$

${ }^{1}$ Department of Radiation Oncology, Hubei Cancer Hospital, Tongji Medical College, Huazhong University of Science and Technology, Wuhan, China, ${ }^{2}$ Department of Oncology, Renmin Hospital of Wuhan University, Wuhan, China, ${ }^{3}$ Department of Cardiology, The First Affiliated Hospital of Chongqing Medical University, Chongqing, China, ${ }^{4}$ Shenzhen Nanshan District People's Hospital, Shenzhen, China, ${ }^{5}$ Department of Vascular Surgery, The Second Affiliated Hospital of Nanchang University, Nanchang, China, ${ }^{6}$ Department of Endocrine, The First Affiliated Hospital of Chongqing Medical University, Chongqing, China, ${ }^{7}$ Department of Cardiology, Hubei Shiyan Taihe Hospital, Shiyan, China

Keywords: schisandrin B, cardiotoxicity, anti-apoptotic pirarubicin, anti-apoptotic, THP

\section{A Corrigendum on}

Schisandrin B Antagonizes Cardiotoxicity Induced by Pirarubicin by Inhibiting Mitochondrial Permeability Transition Pore (mPTP) Opening and Decreasing Cardiomyocyte Apoptosis by Shi, H., Tang, H., Ai, W., Zeng, Q., Yang, H., Zhu, F., Wei, Y., Feng, R., Wen, L., Pu, P., and He, Q. (2021) Front. Pharmacol. 12:733805. doi: 10.3389/fphar.2021.733805

In the original article, there was an error in the Funding section. We neglected to include "National Key R\&D Program of China (Grant numbers: 2018YFC1311400, 2018YFC1311404)".

The authors apologize for this error and state that this does not change the scientific conclusions of the article in any way. The original article has been updated.

Publisher's Note: All claims expressed in this article are solely those of the authors and do not necessarily represent those of their affiliated organizations, or those of the publisher, the editors and the reviewers. Any product that may be evaluated in this article, or claim that may be made by its manufacturer, is not guaranteed or endorsed by the publisher.

Copyright $\odot 2021$ Shi, Tang, Ai, Zeng, Yang, Zhu, Wei, Feng, Wen, Pu and He. This is an open-access article distributed under the terms of the Creative Commons Attribution License (CC BY). The use, distribution or reproduction in other forums is permitted, provided the original author(s) and the copyright owner(s) are credited and that the original publication in this journal is cited, in accordance with accepted academic practice. No use, distribution or reproduction is permitted which does not comply with these terms. 